\title{
Less is more: A novel single-tablet regimen with two-drugs, dolutegravir/lamivudine
}

\author{
Jianjun Sun, Hongzhou Lu* \\ Department of Infection and Immunity, Shanghai Public Health Clinical Center, Shanghai, China.
}

\begin{abstract}
SUMMARY Combined antiretroviral therapy (cART) has significantly reduced human immunodeficiency virus (HIV) associated morbidity and mortality and turned HIV infection into a manageable chronic condition. However, lifelong cART is still required. Two-drug regimens could reduce the number of HIV agents and lower the adverse events caused by lifelong medication. A new two-drug regimen, DEVATO, consisting of dolutegravir and lamivudine has durable efficacy, is well-tolerated, and has a high barrier to viral resistance, which is why it is recommended as a new first-line treatment option for people living with HIV infection.
\end{abstract}

Keywords HIV infection, two-drug regimen, dolutegravir/lamivudine

Combined antiretroviral therapy (cART) has significantly reduced human immunodeficiency virus (HIV) associated morbidity and mortality and today HIV infection has largely become a manageable chronic disease. Without timely cART, however, HIV infection can still progress to acquired immune deficiency syndrome (AIDS) and result in death. People with HIV infection can be treated with cART, which can suppress the level of HIV in the body, control the spread of the virus, and encourage the immune system response to other pathogens.

Based on the achievements of anti-HIV treatments, AIDS-related deaths have been reduced by $64 \%$ since a peak in 2004 and by $47 \%$ since 2010 (1). At the same time, there were 27.5 million people receiving cART in 2020 (1). Along with the aforementioned success of HIV treatments, cART is lifelong and a high level of adherence is required. A particular regimen for a patient with HIV requires consideration of virologic efficacy, toxicity, pill burden, dose frequency, drugdrug interaction potential, HIV resistance test results, comorbidity status, accessibility of medication, and cost. Consequently, the strategy of antiretroviral therapy still needs to be optimized.

As is well known, a classic cART regimen for HIV patients generally consists of two nucleoside/nucleotide reverse transcriptase inhibitors (NRTIs) in combination with a third core antiretroviral drug from one of three drug classes: a non-nucleoside reverse transcriptase inhibitor (NNRTI), a protease inhibitor (PI), or an integrase strand transfer inhibitor (INSTI). In the early days of cART, the available antiretroviral drugs were associated with substantial short-term and long-term adverse events and were not well tolerated (2). Patients were further encumbered by a heavy pill burden. This led to the exploration of an NRTI-sparing treatment strategy: a one- or two-drug regimen that does not include antiretroviral drugs from the NRTI drug class $(3,4)$.

Two-drug regimens (2DRs) have been investigated as an approach to reduce the number of antiretroviral agents taken by individuals receiving lifelong cART $(5,6)$. Previous findings from studies of 2DRs have indicated the efficacy of regimens consisting of lamivudine (3TC) and a core agent with a high barrier to HIV resistance. Dolutegravir (DTG) has demonstrated a high barrier to resistance both in clinical trials and in the real world. DTG and 3TC provide a dual target blockade of the HIV life cycle and have complementary pharmacokinetic (PK) profiles, with matched 24-hour PK and PK tails (intracellular 3TC metabolism). The PADDLE study (7) indicated that $3 \mathrm{TC}$ in combination with DTG had a high potency and efficacy. Two clinical studies, GEMINI-1 and GEMINI-2 (ClinicalTrials.gov identifiers NCT02831673 and NCT02831764, respectively), were identically designed randomized, double-blind, parallelgroup, multi-center, phase III, non-inferiority studies. Participants with HIV RNA $\leq 500,000 \mathrm{c} / \mathrm{mL}$ at screening were randomized in a 1:1 ratio (stratified by plasma HIV RNA and CD4+ cell count) to once-daily treatment with $\mathrm{DTG}+3 \mathrm{TC}$ or DTG + tenofovir/emtricitabine (TDF/ FTC). The primary endpoint was the proportion of participants with plasma HIV RNA $<50 \mathrm{c} / \mathrm{mL}$ at week 48 (8). In the primary analysis of the GEMINI-1 and 
GEMINI-2 studies at week 48, the 2DR regimen DTG $+3 \mathrm{TC}$ was non-inferior to the 3-drug regimen DTG + TDF/FTC in the treatment of cART-naive adults with HIV (8). The DTG +3 TC regimen maintained noninferior efficacy over 96 weeks vs. DTG + TDF/FTC in cART-naive adults, with low rates of confirmed virologic withdrawal (CVW) and no development of resistance in either treatment group (9). DTG + 3TC maintained noninferior efficacy $v s$. DTG + TDF/FTC in ART-naive adults and demonstrated a high barrier to resistance, with low rates of CVW through week 144 (10). Moreover, overall safety and tolerability were comparable between the two groups. There was a lower risk of drug-related adverse effects with DTG + 3TC than with DTG + TDF/ FTC. Changes in renal and bone biomarkers generally favored DTG $+3 \mathrm{TC}$. These results confirmed the durable efficacy, tolerability, and high barrier to resistance of DTG +3 TC, further substantiating the regimen of DTG $+3 \mathrm{TC}$ as a first-line treatment option for people living with HIV infection (PLWH).

In the guidelines for HIV prevention and treatment in both the US and Europe, DTG +3 TC is now recommended as an initial regimen for most PLWH, with exceptions for individuals with HIV RNA > $500,000 \mathrm{c} / \mathrm{mL}$, co-infection with hepatitis B virus (HBV), or in whom therapy is started before the results of HIV genotypic resistance testing for reverse transcriptase or HBV testing are available $(11,12)$.

In addition, the regimen of DTG/3TC is costeffective and would offer significant cost savings $(13,14)$. In addition to the confirmed durable efficacy and tolerability, the lower cost of DTG/3TC would make it a reasonable, and possibly preferred, treatment option for patients who are either treatment-naive or who need a different regimen. Conceivably, the number of HIV patients who could benefit from the DTG/3TC regimen could be substantial.

Funding: The work was supported by the Shanghai Project to Establish Key Clinical Specialties (grant no. shslczdzk01102).

Conflict of Interest: The authors have no conflicts of interest to disclose.

\section{References}

1. UNAIDS. Global HIV \& AIDS statistics-Fact sheet. https://www.unaids.org/en/resources/fact-sheet (accessed July 20, 2021).

2. Nolan D, Mallal S. Complications associated with NRTI therapy: Update on clinical features and possible pathogenic mechanisms. Antivir Ther. 2004; 9:849-863.

3. Canadian Agency for Drugs and Technologies in Health. Clinical Review Report: Dolutegravir/Lamivudine (Dovato): (ViiV Healthcare ULC): Indication: As a complete regimen for the treatment of human immunodeficiency virus type 1 (HIV-1) infection in adults and adolescents 12 years of age and older and weighing at least $40 \mathrm{~kg}$. https://pubmed.ncbi.nlm.nih. gov/31877000/ (accessed July 20, 2021).

4. Riddler SA, Haubrich R, DiRienzo AG, et al. Classsparing regimens for initial treatment of HIV-1 infection. N Engl J Med. 2008; 358:2095-2106.

5. Back D. 2-Drug regimens in HIV treatment: Pharmacological considerations. Germs. 2017; 7:113114.

6. Kelly SG, Nyaku AN, Taiwo BO. Two-drug treatment approaches in HIV: Finally getting somewhere? Drugs. 2016; 76:523-531.

7. Fida M, Rizza SA, Temesgen Z. Dolutegravir plus lamivudine dual therapy - A new option for initial antiretroviral therapy. Drugs Today (Barc). 2019; 55:297304.

8. Cahn P, Madero JS, Arribas JR, et al. Dolutegravir plus lamivudine versus dolutegravir plus tenofovir disoproxil fumarate and emtricitabine in antiretroviral-naive adults with HIV-1 infection (GEMINI-1 and GEMINI-2): Week 48 results from two multicentre, double-blind, randomised, non-inferiority, phase 3 trials. Lancet. 2019; 10167:143-155.

9. Cahn P, Madero JS, Arribas JR, et al. Durable efficacy of dolutegravir plus lamivudine in antiretroviral treatmentnaive adults with HIV-1 infection: 96-week results from the GEMINI-1 and GEMINI-2 randomized clinical trials. J Acquir Immune Defic Syndr. 2020; 83:310-318.

10. Cahn P, Madero JS, Arribas JR, et al. Durable efficacy of dolutegravir (DTG) plus lamivudine (3TC) in antiretroviral treatment-naïve adults with HIV-1 infection: 3-year results from the GEMINI studies. J Int AIDS Soc. 2020 (Suppl. 7). https://onlinelibrary.wiley.com/doi/ epdf/10.1002/jia2.25616 (accessed July 25, 2021).

11. Panel on Antiretroviral Guidelines for Adults and Adolescents. Guidelines for the Use of Antiretroviral Agents in Adults and Adolescents with HIV. Department of Health and Human Services. https://clinicalinfo. hiv.gov/sites/default/files/guidelines/documents/ AdultandAdolescentGL.pdf (accessed July 20, 2021).

12. European AIDS Clinical Society. EACS Guidelines 2020. https://www.eacsociety.org/guidelines/eacs-guidelines/ (accessed July 20, 2021).

13. Butler K, Anderson SJ, Hayward O, Jacob I, Punekar YS, Evitt LA, Oglesby A. Cost-effectiveness and budget impact of dolutegravir/lamivudine for treatment of human immunodeficiency virus (HIV-1) infection in the United States. J Manag Care Spec Pharm. 2021; 27:891-903.

14. Girouard MP, Sax PE, Parker RA, Taiwo B, Freedberg KA, Gulick RM, Weinstein MC, Paltiel AD, Walensky RP. The cost-effectiveness and budget impact of 2-drug dolutegravir-lamivudine regimens for the treatment of HIV infection in the United States. Clin Infect Dis. 2016; 62:784-791.

Received July 22, 2021; Revised July 26, 2021; Accepted July 30, 2021.

\section{*Address correspondence to:}

Hongzhou Lu, Department of Infection and Immunity, Shanghai Public Health Clinical Center, Shanghai, China.

E-mail: luhongzhou@fudan.edu.cn

Released online in J-STAGE as advance publication August 1, 2021. 